\title{
Application of Rank-order Morphological Filtering and Sample Entropy in Feature Extraction of Rotor Fault
}

\author{
Wenbin Zhang \\ Engineering College,Honghe \\ University \\ ECHHU Mengzi, Yunnan, 661100, \\ China \\ Yanjie Zhou \\ Engineering College,Honghe \\ University \\ ECHHU Mengzi, Yunnan, 661100, \\ China
}

\author{
Dewei Guo \\ Engineering College,Honghe \\ University \\ ECHHU Mengzi, Yunnan, 661100, \\ China
}

\author{
Ruijing Teng \\ Department of Mechanical Engineering, \\ Zhejiang University \\ DMEZJU Hangzhou, 310027 , \\ China
}

\author{
Yasong $\mathrm{Pu}$ \\ Engineering College,Honghe \\ University \\ ECHHU Mengzi, Yunnan, 661100, \\ China
}

\author{
Yanping $\mathrm{Su}$ \\ Engineering College,Honghe \\ University \\ ECHHU Mengzi, 661100, China \\ 626516683@qq.com
}

\begin{abstract}
After deeply analyzing the relation between reason and symptom of rotor fault, the sample entropy was introduced into the fault diagnosis field of rotating machinery. Combined with rank-order morphological filtering and sample entropy, a novel feature extraction method was proposed for rotor. Firstly, the line structure element was selected for rank-order morphological filter to denoise the original signal. Secondly, the sample entropy of de-noised signal was calculated, the de-noised signal types were normal, unbalanced, misalignment, oil-film whirl and rubbing. Finally, the sample entropy was served as fault feature to evaluate the different fault condition. Practical results prove that the proposed method is effective on fault diagnosis of rotating machinery.
\end{abstract}

Keywords-rank-order morphological filter; sample entropy; feature extraction; rotor

\section{INTRODUCTION}

Signal processing technology is the core for condition monitoring and fault diagnosis of rotating machinery. Extraction of fault feature parameter is the key for fault diagnosis, which relates the accuracy of fault diagnosis and reliability of early forecast. Mathematical Morphology is a subject concerning with the shape of an object based on set theory and integral geometry [1]. Its filtering idea is based on the geometrical structure of the filtered signals and realized through moving predefined structure element to match and adjust the singular parts of the signals [2]. However, due to the expansibility of the opening transform and the inverse expansibility of the closing transform, the problem of the statistical deviation exists in the open-closing filter and closeopening filter, traditional morphological filter has some shortcomings. It's necessary to improve it in further step. In

This research is financially supported by Scientific Research Foundation (No. 2012C197) of Education Bureau of Yunnan Province and Innovation Experimental Plot Project Fund (No. MS1102) of Honghe University. All the persons involved in the research projects are thanked for their help. Thank you for your help of corresponding author Yanping Su. this paper we describe a subclass of soft morphological filter. This is the class of soft morphological filter in which the hard center of the structure system is the empty set. It's called the rank-order morphological filter. It introduces the circle statistics idea, which could avoid the shortcoming of traditional morphological filter. Literature [3-4] has used the rank-order morphological filter in vibration signal de-noising and rotor axis purification.

Sample entropy is a good tool to evaluate complexity of non-linear time series, compared with other existing non-linear dynamic methods, it has many good characteristic, such as good residence of noise interference, closer agreement with theory for data sets with known probabilistic content. Moreover, sample entropy displays the property of relative consistency in situations where approximate entropy does not [5]. These performances are suitable for rotor fault feature extraction in practice.

Focusing on the practical vibration signal is always interrupted by noises, which can not express the fault feature, we propose a novel approach based on rank-order morphological filter and sample entropy. The proposed method could extract rotor fault feature and distinguish different fault pattern by different sample entropy.

\section{II.BASIC CONCEPTS OF MORPHOLOGICAL TRANSFORM}

A morphological filter is constructed by different morphological transforms. Firstly, several important morphological transforms are introduced.

Dilation and erosion are two basic morphological transforms. While dilation is the transform used to expand the targeted object and shrink the hole, erosion is the transform used to shrink the targeted object and expand the hole. Let $f(x)$ and $g(x)$ denote 1-D input signal and structure element, where $F=\{0,1, \ldots, N-1\}$ and $G=\{0,1, \ldots, M-1\}$ denote sets on 
which signal $f$ and $g$ are defined, $N \geq M$. Dilation and erosion of $f$ and $g$ are thus defined as follows

$$
\begin{aligned}
(f \oplus g)(n)= & \max _{m=0,1, \cdots, M-1}\{f(n-m)+g(m)\} . \\
& (n=0,1, \ldots, N-M) \\
(f \Theta g)(n)= & \min _{m=0,1, \cdots, M-1}\{f(n+m)-g(m)\} . \\
& (n=0,1, \ldots, N+M-2)
\end{aligned}
$$

Where $\oplus$ denotes dilation transform and $\Theta$ denotes erosion transform.

Usually, dilation and erosion are not mutual inverse. They can be combined through cascade connection to form new transforms. If dilation is next to erosion, such cascade transform is an opening transform. The contrary is a closing transform. The transforms can be computed using the following formulas respectively

$$
\begin{aligned}
& (f \circ g)(n)=[f \Theta g \oplus g](n) . \\
& (f \bullet g)(n)=[f \oplus g \Theta g](n) .
\end{aligned}
$$

Where $\circ$ denotes opening transform and $\bullet$ denotes closing transform [6-7]

\section{III.DEFINITION OF RANK-ORDER MORPHOLOGICAL TRANSFROM}

The structure system $[B, r]$ consists of two parameters, the structure element $B$ and the rank selection parameter $r$. The value of $r$ is such that $1 \leq r \leq|B|$, where $|B|$ is the cardinality of $B$ (set-processing and function-set processing) or the size of the region of support of $B$ (function processing).

Let $B$ denote a structure element, $r$ denote a rank selection parameter and $f$ denote input signal. The rank-order dilation of $f$ by $[B, r]$ is defined as:

$$
(f \oplus[B, r](x)=r \text { th largest of }\{f(x+z)+b(z) \mid z \in B\} .
$$

The function processing standard morphological dilation is a specific instance of function processing rank-order dilation and occurs when $r=1$.

The rank-order erosion of $f$ by $[B, r]$ is defined as:

$$
(f \Theta[B, r](x)=r \text { th smallest of }\{f(x+z)-b(z) \mid z \in B\} .
$$

The function processing standard morphological erosion is a specific instance of function processing rank-order erosion and occurs when $r=1$.

The shape and size of the structure elements have a decisive impact on erosion and dilation operations. The shapes of the structure elements are better when they have some convexities, for example the circle and the triangle whose geometric shapes are simple. In addition, the selection of the shapes of structure elements depends on the waveforms maintained after filtering process. Therefore, considering the characters of vibration signal and requirement of engineering calculation, the flat pattern as the simplest type is selected as the structure element to process the data.

According to the characteristic of vibration signal, the de-noising output is coming from the combined rank-order morphological filters constructed as follows [8]:

$$
y(x)=\left\{f^{\oplus}[B, 1 / 2] \Theta[B, 1]+f^{\oplus}[B, 1 / 2] \Theta[B, 0]\right\} / 2 .
$$

Here, $r=1 / 2$ denotes the median filtering for signal [8].

\section{IV.DEFINITION OF SAMPLE ENTROPY}

Let $[x(n)]=x(1), x(2), \ldots, x(N)$ denotes $N$-elements time series representing gear vibration signal. Then, the estimation algorithm of sample entropy is consisted of the following steps [9-10]:

A creating of $m$ vectors defined as:

$$
\begin{gathered}
X_{m}(i)=[x(i), x(i+1), \ldots, x(i+m-1)] . \\
(i=1,2, \ldots, N-m+1) .
\end{gathered}
$$

$B$ calculation of a distance between two vectors in the following way:

$$
d\left[X_{m}(i), X_{m}(j)\right]=\max _{k=0, \cdots, m-1}|x(i+k)-x(j+k)| .
$$

C calculation of number of similar segments in two vectors:

$$
\begin{gathered}
n_{m}=\# d\left[X_{m}(i), \quad X_{m}(j)\right] \leq r, \text { while } i \neq j . \\
n_{m+1}=\# d\left[X_{m+1}(i), \quad X_{m+1}(j)\right] \leq r, \text { while } i \neq j .
\end{gathered}
$$

where $r$ is a tolerance parameter.

D calculation of similarity measures of these segments:

$$
\begin{aligned}
& B_{i}^{m}(r)=\frac{1}{N-m+1} n_{m} . \\
& A_{i}^{m}(r)=\frac{1}{N-m+1} n_{m+1} \quad \text { while } i=1, \ldots, N-m .
\end{aligned}
$$

E calculation of mean measures of the similar signal segments:

$$
B_{m}=\frac{\sum_{i=1}^{N-m} B_{i}^{m}(r)}{N-m} .
$$




$$
A_{m}=\frac{\sum_{i=1}^{N-m} A_{i}^{m}(r)}{N-m} .
$$

F Calculation of sample entropy estimation

$$
\operatorname{SampEn}(m, r)=-\operatorname{In} \frac{A^{m}(r)}{B^{m}(r)}
$$

\section{V.PRACTICAL APPLICATION}

In order to verify good effectiveness in fault feature extraction, all vibration signals were collected from the experimental testing of rotor. The speed of the motor is 3000 RPM and the sample frequency is $1024 \mathrm{~Hz}$. The five conditions were tested that were normal, unbalanced, misalignment, oil-film whirl and rubbing. Now we get fifteen sampled data sets of each condition. First, we use rank-order morphological filter to remove noise interference before further procedure. Let's take the normal condition and rubbing signals for example, Figure 1 and Figure 2 show the de-noising results in time and frequency domain.

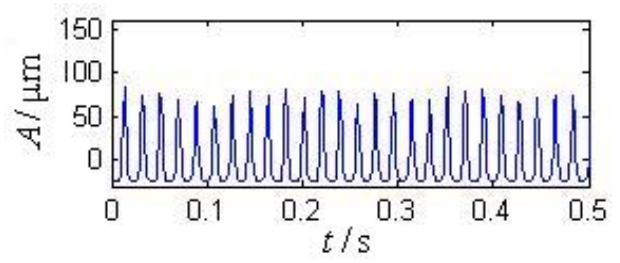

(a) Original waveform of normal signal.

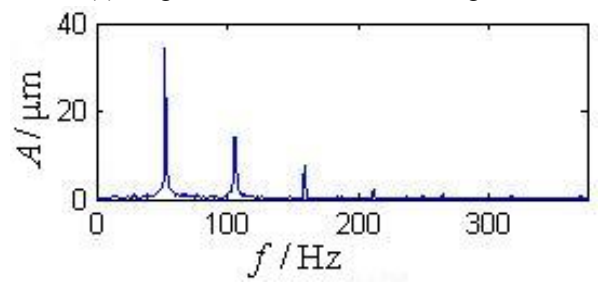

(b) Spectrum of original normal signal.

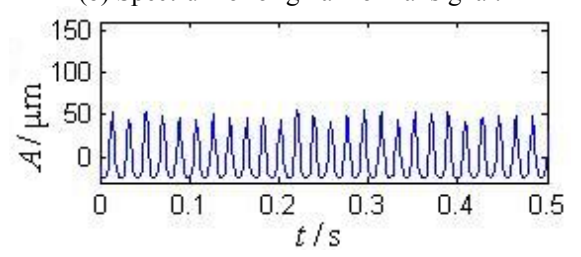

(c)Waveform of normal signal de-noised by rank-order morphological filter.

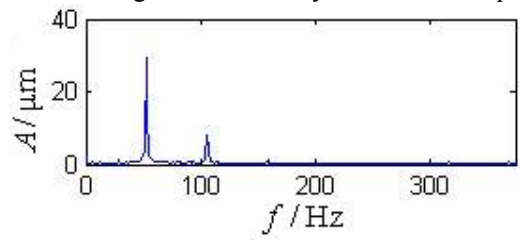

(d)Spectrum of normal signal de-noised by rank-order morphological filter.

Figure 1. Waveforms in time and frequency domain of normal signal before and after de-noised by rank-order morphological filter.

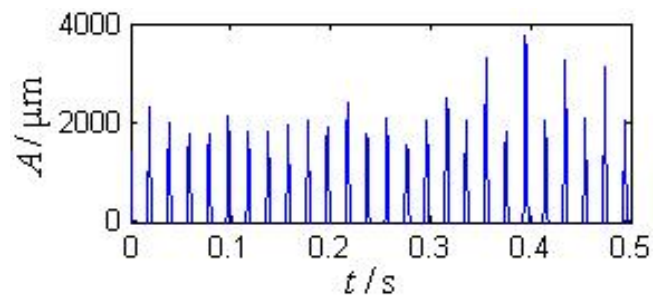

(a) Original waveform of rubbing signal.

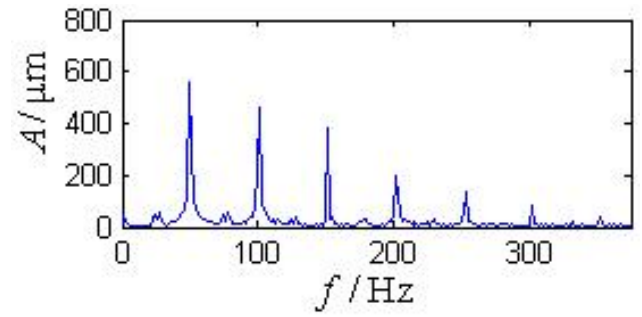

(b) Spectrum of original rubbing signal.

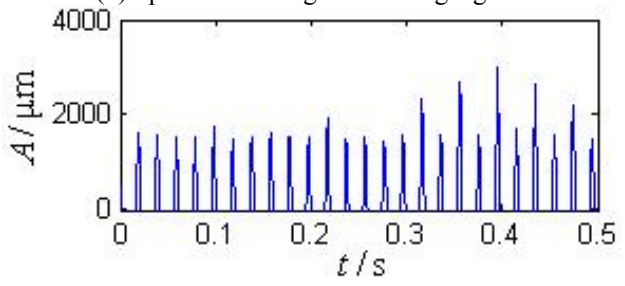

(c)Waveform of rubbing signal de-noised by rank-order morphological filter.

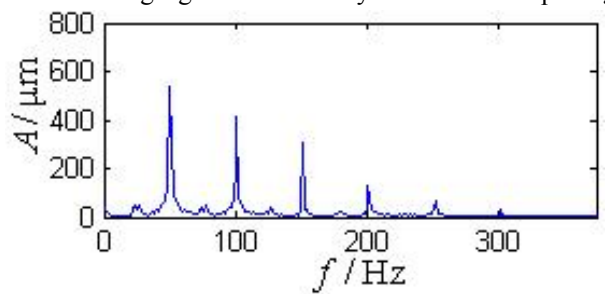

(d)Spectrum of rubbing signal de-noised by rank-order morphological filter.

Figure 2. Waveforms in time and frequency domain of rubbing signal before and after de-noised by rank-order morphological filter.

Comparing the above two figures, we can see that peak pulse and noise interference are removed clearly, the fault feature is obtained obviously. It is very useful in the next procedure.

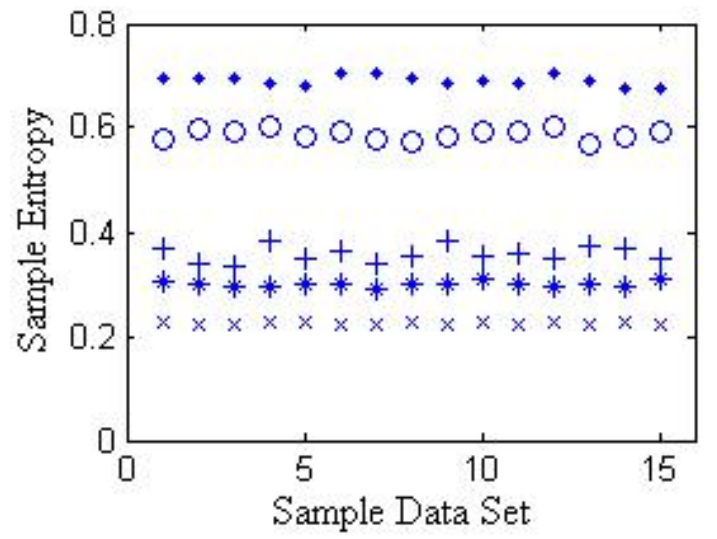

Figure 3. Sample entropy of five fault patterns after rank-morphological filter de-noising 
Next, we calculate the sample entropy of each sample data set. Figure 3 shows the calculating values of sample entropy from different fault pattern. We can see that different fault pattern correspondence with different sample entropy. So we can diagnosis rotor fault by such feature.

Here, "." denotes sample entropy of normal signal, "O" denotes sample entropy of unbalanced signal, "*” denotes sample entropy of misalignment signal, "+" denotes sample entropy of oil-film whirl signal and " $x$ " denotes sample entropy of rubbing signal .

Figure 4 shows the sample entropy of original signal, we know that sample entropy from non-denoised signal can not distinguish different fault pattern. It is improved the importance of de-noising.

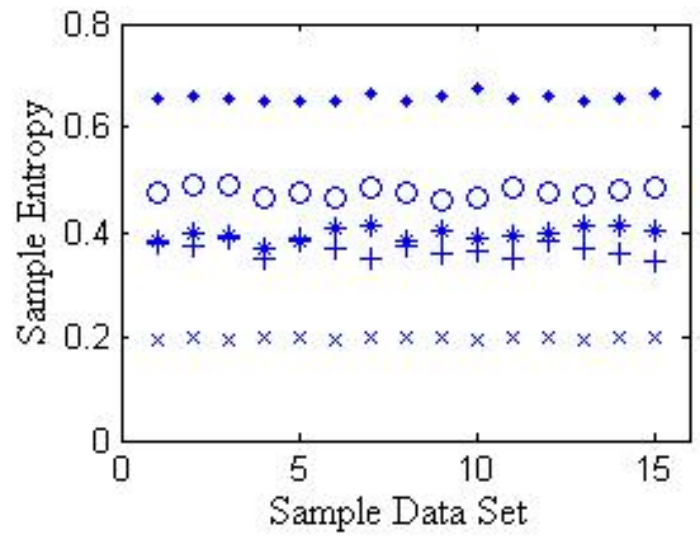

Figure 4. Sample entropy of five fault patterns before rank-morphological filter de-noising

\section{VI.CONCLUSION}

In this paper, we propose a new fault extraction method by using rank-order morphological filter and sample entropy. The rank-order morphological filter could eliminate noise interference before the further process of the sample data. And the sample entropy could serve as a good feature parameter for different rotor fault. Practical results show the good performance of the proposed method in feature extraction of rotor fault. It will supply a new method for fault diagnosis of rotating machinery.

\section{REFERENCES}

[1] Q. M. Zhang and H. J. Liu, "Processing of sampled power system data based on generalized morphological filtering", Electric Power Automation Equipment, 2006, Vol. 26, pp. 45-48.

[2] Maragos. P. and Schafer R.W., "Morphological Filters-Part II: Their Relations to Median, Order-Statistics, and Stack Filters", IEEE Trans. Acoustics, Speech and Signal Processing, 1987, Vol. 35, pp. 1170-1184.

[3] W. B. Zhang, H. J. Wang and R. J. Teng, "Application of rank-order morphological filter in vibration signal de-noising", Proceedings-2010 3rd International Congress on Image and Signal Processing, Yantai, China, 2010, vol.8, pp.4025-4027.

[4] W. B. Zhang, Y. P. Su, Y. J. Zhou, R. J. Teng and S. K. $\mathrm{Xu}$, "Application of rank-order morphological filter in refinement of rotor center's orbit", Proceedings-4th International Congress on Image and Signal Processing, Shanghai, China, 2011, vol.4, pp.2278-2280.

[5] D. E. Lake, J. S. Richman, M. P. Geriffin and J. R. Moorman, "Sample entropy analysis of neonatal heart rate variability", Am J Physiol Regul Integr Comp Physiol, 2002, vol. 283,pp.789-797.

[6] W. B. Zhang, X. J. Zhou and Y. Lin, "Application of generalized morphological filter in vibration signal processing", Transactions of the CSAE, 2008, Vol.24, pp.203-205.'

[7] W. B. Zhang, H. J. Wang, L. Shen, Q. Cai and J. S. Li, "Purification of axis trace based on adaptive generalized morphological filter", Proceedings-2nd International Congress on Image and Signal Processing, Tianjin, China, 2009, Vol.8, pp.4105-4109.

[8] Neal R. H. and Stephen M., "Rank-order morphological filters: a new class of filters", In IEEE Workshop on nonlinear signal and image conference, 1994.

[9] Y. G. Luo and C. Z. Chen, "Diagnostic analysis of grey network in rotary machinery trouble," Compressor Blower \& Fan Technology, 2001, vol.4, pp.38-40.

[10] Y. B. Dong and X. L. Zhang, "Determination method for identification coefficient of grey relational grade and applying in mechanical faults diagnosis," Equipment Manufacturing Technology, 2008, vol. 3, pp.121-122, 125. 